\title{
Research on Construction Practice of Assembled Substation-A Case in Anhui Xuancheng Xinhang 110kV Substation Project
}

\author{
Chen Ximing ${ }^{1}$, Bao Dian ${ }^{2}$, Qian Chaojun ${ }^{3}$, Zhang Shao-jie ${ }^{4}$ \\ ${ }^{1}$ Anhui Electric Power Engineering Supervision Co., Ltd., Hefei, Anhui, 230071, P.R. China; \\ ${ }^{2}$ State Grid XuanCheng Electric Power Co., Ltd., Xuancheng, Anhui, 242000, P.R. China; \\ ${ }^{3}$ State Grid Anhui Electric Power Co., Ltd. Construction Company, Hefei, Anhui, 230071, P.R. China; \\ ${ }^{4}$ Anhui Jianzhu University, BIM Engineering Center of Anhui Province, Hefei, Anhui, 230601, P.R. China;
}

\begin{abstract}
The assembled substation is a substation construction that is "resource-saving, environment-friendly, and industrialized" through prefabrication and on-site installation. Taking the Xuancheng Xinhang $110 \mathrm{kV}$ substation project in Anhui as an example, this paper studies the standardization development of assembled industrial buildings and substations at home and abroad, points out the problems existing in the construction and building practice of the assembled substation, and puts forward relevant suggestions for these problems.
\end{abstract}

\section{Introduction}

[2013 No. 1 Document State Council] proposes: "Accelerating the research and development of green building related technologies, vigorously developing green building materials, and promoting Building industrialization", the State Grid Corporation actively responded and proposed the "two-type one" and "two-type three new" strategies for power grid construction. The assembled substation is the product of national policy guidance and the development of the national grid strategy. The construction of its project management and control system is also the need to deepen the implementation of the relevant guidance documents.

\section{Overview of the development of standardization of assembled industrial buildings and substations at home and abroad}

\subsection{Design and construction of assembled buildings}

The assembly integral construction drawing design system is based on the conventional clear water concrete, and the component detailed design is carried out, and the information of the water and electricity reserve pre-buried is integrated into each component drawing, produced at the assembled component factory and after reaching the strength, transported to the concrete structure for assembly and assembly, and assembled into a monolithic structure equivalent to cast-in-place.

The assembled building mainly has the following technical features: 1 Design standardization: In the design stage, combined with the technical characteristics of the assembled building, the overall structure is reasonably disassembled into individual components, the shape and size of the components are optimized, and the repeating of the prefabricated mold is improved. 2 Production standardization and integration: Prefabricated component factories produce components under standard operating procedures, which are not affected by external environments such as seasons and weather, and have high production efficiency. 3 Mechanized construction and construction speed: The assembled building adopts mechanized assembly construction, and the installation speed is fast. The dry construction is the main method, which reduces a large number of temporary measures and wet work such as on-site plastering, which can greatly speed up the construction. 4 materials to reduce consumption, assembled building is less: Prefabricated buildings can save $5 \%$, save $70 \%$, save $40 \%$, and reduce construction waste by $80 \%$.

\subsection{Design and construction of assembled substation}

Assembled substations mainly have the following technical characteristics: 1 design standardization 2 production standardization, integration 3 mechanized construction, construction speed is fast 4 materials to reduce consumption, assembled building is less.

Electricity as the first source of energy also needs to develop rapidly. There are more and more power-related 
supporting buildings, and they are widely distributed in urban centers. In the process of substation construction, the operating conditions are extremely poor, the construction site is narrow, and the contradiction between quality, construction period and cost is prominent. More and more substation construction conditions and construction period requirements are consistent with the technical characteristics of the assembled buildings. Therefore, the promotion of assembled buildings in the power industry has extremely high social and economic benefits.

\subsection{Introduction to the development of standardized design of foreign substation}

Compared with developed countries, the penetration rate of assembled buildings in China is huge. Developed countries with high levels of industrialization have developed various types of assembled building-specific systems. Such as the British L-board system, the French pre-stressed assembly frame system, the German prefabricated hollow formwork wall system, the US prefabricated parking garage system, and the Japanese multi-layer assembled collective housing system. In 2017 , the penetration rate of assembled buildings in the United States, Japan, France, Singapore, etc. reached or exceeded $70 \%$. Compared with China's penetration rate of $8.4 \%$ in 2017 , China's assembled building penetration rate is large, and the room for improvement is huge.

\subsection{The core problems in the design of domestic assembled substation}

Assembled substations are currently used less in the grid system and are not universally applicable. In China, assembled structures are used in substation design and are rarely used in commercial and construction sites. In civil buildings, prefabricated exterior siding, stairs and slabs have been widely used for their unique advantages. However, it is rarely used in industrial buildings, accounting for less than $1 \%$. In the past, assembled structures were used in power grids and were rarely used in technical specifications.

At present, there are very few assembled structures in substation construction. The main problems are as follows: 1 Assembled construction is a systematic project, and there are currently few units with assembled industrial building, production and construction experience. The construction bidding can be selected in a small scope, and the civil construction and installation may need to be divided into development kits, and the bidding restrictions are more. 2 Because the substation belongs to industrial buildings, the room size of equipment varies with the layout of the building. The height of the building is higher than that of the civil building. The production and installation of the components require a large number of molds and special tools. A single project cannot complete the amortization and the single project can increase by $15 \% \sim 20 \%$. In the case of a small amount of component manufacturing, there is no obvious advantage in economic comparison. 3
The design workload is large, because there are a large number of process equipment installed in the substation, and there are many holes and structures required for the embedded parts and structures. However, once the prefabricated structure is completed, there is no possibility of change, so the equipment order must be completed and confirmed. After the technical regulations are arranged, the civil construction work is carried out. The design cycle is relatively long, and a large number of large-scale components are required to be completed. The semi-finished products of the prefabricated components are subjected to two-stage verification in the state of mold-cutting, transportation and hoisting processes, so the ratio is larger. The conventional design requires an increase of $100 \%$ of the workload and a $40 \%$ design period. However, because of the unique advantages of assembly, with the continuous trial and progress of technology, it will gradually replace the traditional structural mode.

\section{Standardized design and construction case study of assembled substation}

\subsection{Project Engineering Overview}

The communication project of the Xuancheng Xinhang $110 \mathrm{kV}$ substation in Anhui Province is located in Xuancheng City. The project was invested and constructed by the State Grid Anhui Power Company, with a project capital of $20 \%$. The rest of the funds were raised in the form of loans for project construction. The project is mainly divided into (1) major production projects, including major production buildings, power distribution equipment buildings and water supply system buildings; (2) auxiliary production projects, including station buildings and special structures; (3) individual projects related to substation, including ground handling and off-street roads.

\subsection{Some key construction problems of assembled substation}

\subsubsection{Assembled wall}

The assembled wall has a fast construction schedule, no need for wall painting and decoration, reducing on-site construction waste, and improving the safety and civilized construction. The disadvantage is that the board is easily deformed and cracked, and the thickness is inconsistent, and the seam is relatively large. The reason for this problem is that the prefabrication accuracy of the plate is poor.

\subsubsection{Integrated wallboard for power distribution room}

The advantages of the integrated wall panel of the power distribution unit in the project are as follows: the construction is convenient and quick, and the construction process of the brickwork, steel bars and 
formwork is reduced. Because it is a molding, it reduces the construction waste generated during the decoration process. The disadvantage is that the prefabrication accuracy of the integrated wallboard is poor, and the gap between the wall panels is too large during the construction process, and the seams of the wall panels are inconsistent (Figure 2). During the construction process, it is also found that the strength of the wallboard is not as good as color steel plate. Based on the above construction problems, the conclusion is that in the process of integrated wallboard production, the design should communicate well with the manufacturer, reserve the dark application pipeline, and improve the precision of prefabrication.

\subsubsection{Accident oil tank, septic tank}

In the assembled substation, the accident oil pool and septic tank also have convenient and quick construction, reduce the brick masonry, steel bar and formwork construction process, and form one time, which reduces the advantages of construction garbage generated during the decoration and decoration process. The disadvantage is that the reinforced concrete material is too cumbersome to make the lifting problem difficult. Accident oil pools and septic tanks are generally assembled on site and assembled in three parts. The main problem is that the joints are not easy to handle and the lifting points are unreasonable. Therefore, when constructing a substation, the septic tank is recommended to use a finished glass slag septic tank; at the same time, the lifting point should be placed outside the oil sump, and the waterproofing scheme of assembling the splicing joint should be considered.

\subsubsection{Steel structure integrated wallboard firewall}

During the construction of the assembled substation, the steel structure integrated wall panel firewall also has the advantages of convenient and quick construction, reducing the advantages of steel bar, formwork and concrete construction process; the disadvantage is that the fire resistance of the steel structure skeleton is not as good as the traditional concrete structure.

\subsection{Problems in the construction of assembled substation and Suggestions for improvement.}

During the construction of Xuancheng Xinhang $110 \mathrm{kV}$ substation project in Anhui, it was found that there were problems in the procurement, supply and after-sales of prefabricated parts, the protection of finished products during transportation, the processing precision of prefabricated components, and the stacking of materials on site, is summarized as follows:

(1) The manufacturer of the wall prefabricated parts purchased by the project is Wuhu Jianhua Building Materials. According to the requirements of the market enterprise operation, the contract must be in accordance with the requirements of the other party, without leaving the quality guarantee, and it is the payment to the delivery. This type of operation makes it difficult to control the delivery speed and quality of the prefabricated parts, and it is difficult to ensure after-sales service (Figure 1).

(2) During the transportation of concrete prefabricated parts, there are also problems such as serious superposition of prefabricated components, and the protection of finished products is not in place, and the bumping is serious. The economic transportation distance of concrete prefabricated parts is less than 200 kilometers, but in order to save freight during transportation, it is often loaded with more concrete prefabricated parts, and the transportation speed is fast, which causes damage to concrete prefabricated parts.

In addition, the wall panel of the arrival wall also has a fracture phenomenon.

(3) During the on-site construction and construction process, the fenced wind-resistant column of the arrival is difficult due to the low processing precision, the vertical is not vertical and there is no lifting point. Moreover, due to the horizontal placement of the steel formwork in the concrete pouring method, many air bubbles are present at the groove. After on-site analysis, it is necessary to set safe and reliable lifting points at the top of the column. At the same time, the column formwork is poured in vertical pouring to eliminate bubbles and reduce subsequent treatment.

(4) Due to the poor process of the wall member clear water raft, the manufacturer has applied the protective liquid treatment on the outer surface, resulting in thickening of the wall panel, and the size of the wind-resistant column slot is reduced, thus making the installation of the wall panel difficult, many wall panels The thickness does not match the slot size, which wastes a lot of mechanical work and manpower. For the template processing accuracy is poor, the concrete ratio cannot meet the requirements of fair-faced concrete, resulting in poor finished products, the defects must be treated and then brushed with clear water concrete protection fluid, but this also causes the cross-section size of the preform changes, which is installed bring difficulties.

(5) The design elevation of the wall of the wall is the positive and negative of the station area. The current elevation of the surrounding wall of the new Hangchang project is lower than the design elevation of the site. The backfill of the wall at the bottom of the wall will be washed away by rain and sedimentation, which will lead to holes at the bottom. And small animals outside the station may enter the station. There is no drainage hole in the wall of the station area, which increases the severity of rain erosion.

(6) In the wall drawing design, the height of the top wall is the same as the height of the top of the column. The top is directly embedded in the wall. The column cap is also directly embedded in the top of the column, and the depth is $1 \mathrm{~cm}$. However, there are two problems at the scene: 1) The left and right sides of the column cap are located on the wall panel. Although there are grooves in the column cap, there are no grooves on the left and right sides, so the column head cannot be directly embedded in the column cap. 2) The manufacturer did not consider the 
cumulative error of the wallboard stacking when producing the wallboard, resulting in the top panel height of the wallboard being 1-3 cm higher than the top of the pillar after the installation of the five wallboards. It is recommended that in subsequent designs, the cap and the top are not at an elevation to avoid installation on site.

(7) The wall panel and the top design are connected by two bolts, and the mounting holes are reserved. Due to the processing accuracy, the mounting holes and the bolt holes embedded in the wall panel cannot be completely matched, and only one bolt can be installed for each pressure top.

(8) The prefabricated cable trench is convenient for demoulding. There are 4 rebar hooks at the bottom, and the hook protrudes from the bottom plate, which makes the installation flatness uncontrollable. It is necessary to cut the hook before installation. It is recommended to set the groove type lifting hole.

(9) Because it is a custom steel mold, the bottom drain groove has no flooding slope, and there is a hidden danger of water accumulation at the bottom of the cable trench. The prefabricated cable trench process is poor, and the thickness of the plate is deviated, resulting in different heights at the top of the installation after forming, and the flatness of the later cover is poor. There is a drainage ditch at the bottom of the cable trench, which causes the thickness to decrease here, so the drainage groove is weak, and this part is easily broken when hoisting. It is recommended to add a drain to avoid water accumulation in the cable trench, and at the same time adjust the cable trench mold, and reserve a certain flood gradient during the concrete pouring process to solve the drainage in the cable trench. Optimize the reinforcement of the cable trench bottom plate to avoid the stress concentration causing the cable trench to break (Figure 2).

(10) There is a certain error in the cooperation between the integrated wall panel and the machining accuracy of the steel structure, which cannot meet the requirements of the design and the drawing error is controlled within $2 \mathrm{~mm}$. At present, the cumulative error caused by on-site processing and installation seriously exceeds the design tolerance, which makes installation on site difficult. According to the actual installation situation at the site, it is still not possible to completely prevent the fire from moving, and the position of the mounting hole still needs to be properly adjusted.

(11) Adequate working time, space and conditions should be given before wall panel installation. Before the installation of the integrated wallboard, the following conditions should be met: the building's scattered water slope and indoor need to be hardened to facilitate the erection of the movable frame; the roof concrete and waterproof need to be completed, to prevent the wall from being polluted when the concrete is poured on the roof and to facilitate the installation of the daughter wall panel Before the installation of the wallboard, the indoor cable trench and the floor should be completed, and the indoor operation should be reduced as much as possible to prevent damage to the construction and pollution of the wall. This can effectively avoid the situation where the construction on site is more chaotic and the safety risk increases.

(12) Since the steel structure of the whole station is bolted, the connecting plate at the top of the roof is higher than the frame beam, and after the installation of the floor board is completed, the gap is exposed here to affect the overall look and feel.

(13) There are many finished components and auxiliary construction parts used in the assembled substation station, and these materials have high requirements on the stacking site, such as: integrated wall panels, various composite panels and plates, precast concrete components, etc. When the peak operation is carried out on site, there is basically no suitable site for material stacking in the station, and there is a problem of secondary transportation when stacked outside the station. Therefore, material stacking on site is also a problem to be solved.

(14) The septic tank is made of reinforced concrete material, which is too cumbersome, and the safety of lifting on site is high, and the installation work is difficult It is recommended to design the finished glass septic tank in the future, which is light and easy to install, and has a good impression (Figure 3 ).

(15) The heaviest weight component (base) of the oil pool weighs 16 tons. After being transported to the site, it is more difficult and safe to unload with a 35-ton crane. The on-site installation uses a 70-ton crane. The design drawings of the accident oil pool are assembled in three parts. Due to the small number of components, the weight of the unit is large. Since it is a fabricated accident oil pool, it is recommended to prefabricate the assembled components more to reduce the weight of the single components. On-site lifting and operation risks, and at the same time, it is convenient to unify the selection of on-site cranes (Figure 4).

(16) It is recommended that the post-assembly wall adopts steel composite wall panel wall, the wall foundation is pre-embedded with bolts, the wind-resistant column is steel structure, and the wall and column piers are composite wall panels. This kind of wall has good perception and easy installation on site.

The first application of the integrated composite wall also showed a slight flaw in the installation process. Since the wall panel is directly transported to the site after production, the rivet position of the exterior wall decorative panel is fixed and uniform. Although the large module has a fine-tuned reserved size during the field installation, it cannot be horizontally and vertically. Moreover, the wall panel will cause damage and pollution of the exterior wall decorative panel during transportation and installation. It is recommended that the decorative panel of such engineering will not be installed first, and it will be installed before the outer wall is glued after the overall installation. The post-installation can effectively control the rivet and the splicing seam to be horizontal and vertical, and the appearance effect is improved significantly. 


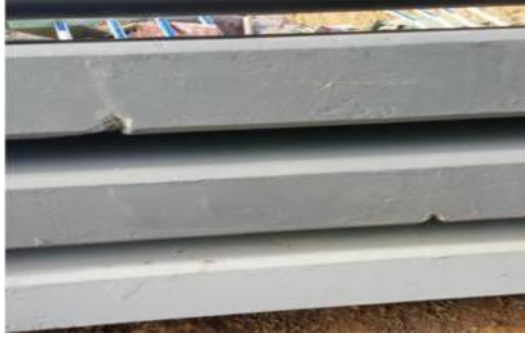

Figure 1. Prefabricated fracture parts

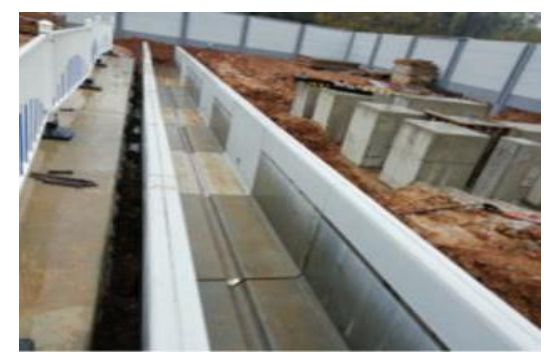

Figure 2. Cable ditch water damaged

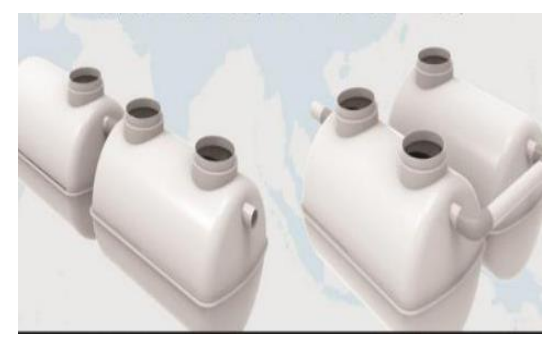

Figure3. Glass tempered septic tank
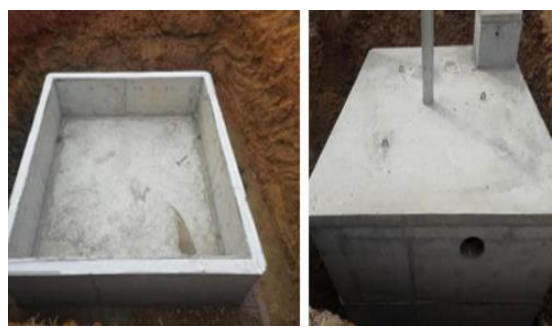

Figure4.Oil pool installation

\section{Conclusion}

Anhui Xuancheng Xinhang 110kV Substation Project is a pilot project for the assembly of substation in Anhui Province in 2019. The project had built a "four-in-one" assembled substation project management and control system, highlighting the essential characteristics of substation "industrialization" and forming targeted, the scientific and perfect project construction management materials provide strong support for the formation of Anhui's unique assembly-type substation construction mode.

\section{References}

1. Chen wenjie. Modular research on $110 \mathrm{kv}$ standard assembled substation $[\mathrm{J}]$. Electric power and energy, 2017, 38 (6): 698-701
2. Wu chengquan. Research and application of $110 \mathrm{kV}$ substation assembled wall [J]. China new technology and new products,2017(21): 70-71

3. Wuchengquan. Application of $110 \mathrm{kV}$ standard distributed smart substation project and prefabricated buildings $[\mathrm{J}]$. China strategic emerging industry, 2017(36):139,141

4. Mao shicheng. Construction practice of $110 \mathrm{kV}$ fully prefabricated substation [J]. Architectural design, 2019 (46): 26-27

5. Ye jianfeng. Construction practice of $110 \mathrm{kV}$ fully prefabricated substation [J]. Power supply and consumption, 2010 (27): 42-45

6. Deng zixing. Based on exploration of prefabricated substation and analysis of its installation technology [J]. Low-carbon world.2016(09) 